\title{
Stacked Tri-Band Circularly Polarized Microstrip Patch Antenna for CNSS Applications
}

\author{
Ding Kang,YU Tong-bin , GUAN Dong-fang \\ Communication Institute of PLA University of Science \\ and Technology \\ Nanjing, China \\ e-mail: dingkang19881203@163.com
}

\author{
Peng Cheng \\ Defense Information Academy \\ Wuhan, China \\ e-mail: raul0421@126.com
}

\begin{abstract}
This paper proposes a novel stacked tri-band circularly polarized antenna which has three independent ports. To obtain tri-band operation, a stacked three layers of microstrip antenna working at different frequency is presented. Each of them is fed by dual feed probes. The simulated results show that the antenna can cover Compass Navigation Satellite System CNSS B3 1.268 GHz, L band 1.615 GHz and S band 2.49 GHz. The proposed antenna has achieved a bandwidth of $3.1 \%, 6.8 \%$ and $2.3 \%$ at each band, respectively. It exhibits small axial ratio under $3 \mathrm{~dB}$ in three bands for the CNSS applications. Details of the proposed antenna design and results for the obtained tri-band circularly polarized performances are presented and discussed.
\end{abstract}

Keywords- tri-band; circularly polarized; CNSS; microstrip patch antenna; stacked.

\section{INTRODUCTION}

With the rapid development of navigation system , many new applications of the wireless communications and sensor systems devices have been growing dramatically. In China, Compass Navigation Satellite System (CNSS), or "BeiDou" in its Chinese name, began to provide navigation and positioning services in the latest years. According to Chinese officials, the CNSS can cover three frequency band , $1258-1278 \mathrm{MHz}, 1610-1626 \mathrm{MHz}$ and $2483-2500 \mathrm{MHz}$. Thus, an antenna which can operate at all of the CNSS frequency is required.

The microstrip antenna has the advantages of low profile, lightweight and low fabrication cost and ease of fabrication [1]. So it is obvious that microstrip antenna has been widely used in communication system. In practice, a circularly polarized (CP) microstrip antenna is needed for the antenna to stably receive signal especially for multi-band CP antenna. In order to achieve multi-band CP antenna, many scholars have done a lot of research. Various types of designs related to multi-band $\mathrm{CP}$ antennas have been reported [3]-[10]. CP antennas have been achieved using both single and dual feed techniques. A single-fed CP antenna has the disadvantages of both narrow AR bandwidth and impedance bandwidth [2], so it is necessary to adopt multi-feed techniques [3]. And recently stacked microstrip antennas have been developed to obtain dualband or tri-band radiation. Some dual-band CP stacked microstrip patch antennas were achieved, like using an aperture couple feed [4] [5], by two different types of stacked patched [6], and by carving an asymmetric S-shaped slot [7]. In [8], a stacked patch technique with a high permittivity dielectric material is used to achieve a compact triple band antenna design. In [9], a three-layer stacked single feed CP microstrip antenna was designed to achieve tri-band CP radiation. Also, a tri-band CP stacked design was introduced in [10] for GPS and CNSS applications. The CP radiations were achieved by inserting the two pairs of narrow slots parallel to the edges of the top square patch and cutting a slit in the bottom square patch.

In this paper, a three ports tri-band CP stacked microstrip antenna for CNSS applications is presented. The tri-band characteristic is achieved by three stacked square patch, and each band of CP radiation is achieved by two probes feeding. This can be realized by use a 90-deg hybrid coupler to generate two orthogonal modes of the patch for the circularly polarized. So three independent ports working with different sense of CP performance is realized by adjusting the feeding location. The proposed antenna can find useful application in "BeiDou" $2^{\text {nd }}$ generation satellite system.

\section{ANTENNA DESIGN}

The desired CNSS antenna must have the following specifications:

1. Multiband operation (B3: $1.268 \mathrm{GHz}$; L: $1.615 \mathrm{GHz}$; S: 2.49GHz);

2. RHCP radiation for B3 and S band, LHCP radiation for $\mathrm{L}$ band, Gain greater than $2.5 \mathrm{dBi}$;

3. Axial ratio (AR) is less than $3 \mathrm{~dB}$ at the frequency (B3/L/S);

4. Feed matched to $50 \Omega$;

The geometry of the proposed stacked tri-band CP microstrip antenna for CNSS applications is shown in Fig. 1

The antenna is made up of three square patches stacked on one another. The three square patches are etched on separate substrates which have the thickness of $h_{1}, h_{2}, h_{3}$, .In order to reduce the geometry of antenna patch, we choose relative permittivity $\varepsilon_{1}=3.5, \varepsilon_{2}=6, \varepsilon_{3}=10$. And a substrate made of Rogers 5880 material ( $\varepsilon_{4}=2.2$ ) has been chosen to accommodate the feeding network. The top, middle and bottom square patches have different side length of $L_{i}$ respectively. 


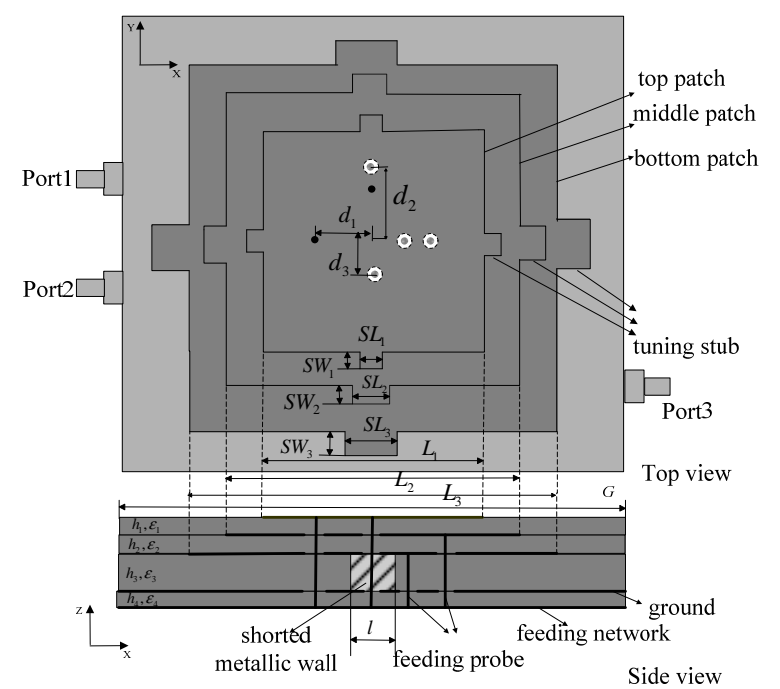

Figure 1. Geometry of the proposed stacked tri-band CP antenna.

In general, the side length is usually about halfwavelength, that is

$$
L_{i}=\frac{c}{2 \sqrt{\varepsilon_{e} f_{i}}}
$$

Where $i=1,2,3, C$ is the speed of the light, $\mathcal{E}_{e}$ is the approximated effective dielectric constant, and $f_{i}$ is the free space resonant frequency of the antenna. Each patch is excited by two probes feeding, which are connected to the patch through two via holes in the other patch. To optimize the antenna performance, four tuning stubs were added to the patch. Experiments shows the shortening of either stub will decrease the effective electromagnetic length of the dominant mode cavity formed by the patch, thereby increasing its resonant frequency. In order to improve the gain at $1.268 \mathrm{GHz}$, a $3 \times 3 \mathrm{~mm}$ square hole was cut in the bottom substrate which add a shorted metallic wall around it. Owing to the adding of the shorted metallic wall, the gain at low frequency can increase about $1 \mathrm{~dB}$, thus it can satisfy the design

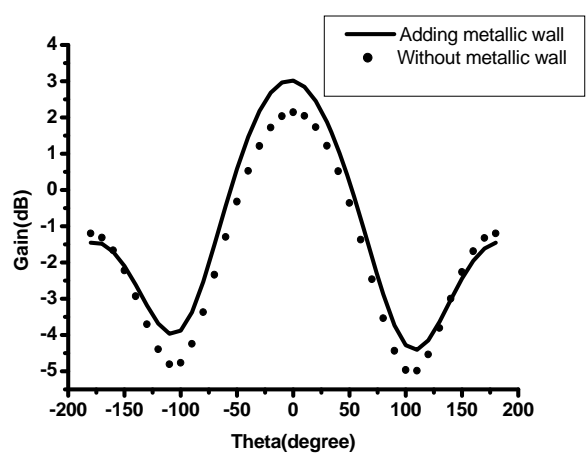

Figure 2. The comparison of gain with different structure at $1.268 \mathrm{GHz}$
The antenna feeding network has to provide 90-deg phase difference between two probes at each band. To achieve this, a compact wideband 90-deg hybrid coupler has been used to supply two probes with balanced signals of equal magnitude and 90-deg phase difference. For the feed position at port 1 and port 3, right-hand CP (RHCP) operation is obtained. Through adjust the phase difference between the two probes, left-hand CP (LHCP) operation is obtained by feeding the patch at port 2. Hence, a trifrequency circularly-polarized antenna is achieved by choosing the different feed port on the antenna. The final dimensions of the parameters are listed in Table I. The proposed antenna is fabricated and measured. The overall ground plane size is $50 \times 50 \mathrm{~mm}$.

TABLE I Dimensions of The Proposed Antenna

\begin{tabular}{c|c|c|c|c|c}
\hline parameter & value & parameter & value & parameter & value \\
\hline \hline$h_{1}$ & $3 \mathrm{~mm}$ & $L_{3}$ & $34 \mathrm{~mm}$ & $S W_{3}$ & $2 \mathrm{~mm}$ \\
\hline$h_{2}$ & $2 \mathrm{~mm}$ & $d_{1}$ & $5.5 \mathrm{~mm}$ & $S L_{1}$ & $1 \mathrm{~mm}$ \\
\hline$h_{3}$ & $2 \mathrm{~mm}$ & $d_{2}$ & $10 \mathrm{~mm}$ & $S L_{2}$ & $4 \mathrm{~mm}$ \\
\hline$h_{4}$ & $1 \mathrm{~mm}$ & $d_{3}$ & $7.2 \mathrm{~mm}$ & $S L_{3}$ & $5 \mathrm{~mm}$ \\
\hline$L_{1}$ & $31 \mathrm{~mm}$ & $S W_{1}$ & $2 \mathrm{~mm}$ & $l$ & $3 \mathrm{~mm}$ \\
\hline$L_{2}$ & $33.5 \mathrm{~mm}$ & $S W_{2}$ & $5 \mathrm{~mm}$ & & \\
\hline
\end{tabular}

\section{MEASUREMENT RESUltS AND DISCUSSIONS}

The characteristics of the proposed tri-band CP antenna are simulated by software Ansoft HFSS 10. The reflection coefficients are measured using a vector network analyzer, and radiation characteristics are measured in the anechoic chamber. The reflection coefficient, axial radio (AR), and radiation patterns measured at the three feeding ports of the antenna are presented in Fig.3,4,and 5, along with the simulated results.

Fig.3 shows the return loss of different port which indicates that the three bands are: $1.268 \mathrm{GHz}, 1.615 \mathrm{GHz}$ and $2.49 \mathrm{GHz}$. A good agreement is obtained between simulation and measurement. The measured impedance bandwidths ( $S_{11}<-10$ ) are $3.15 \%$ with respect to $1.268 \mathrm{GHz}$ (from $1.25 \mathrm{GHz}$ to $1.29 \mathrm{GHz}$ ) for the low band, 6.3\% with respect to $1.615 \mathrm{GHz}$ (from $1.53 \mathrm{GHz}$ to $1.63 \mathrm{GHz}$ ) for the middle band and $2.8 \%$ with respect to $2.49 \mathrm{GHz}$ from 2.47 $\mathrm{GHz}$ to $2.54 \mathrm{GHz}$ for the upper band.

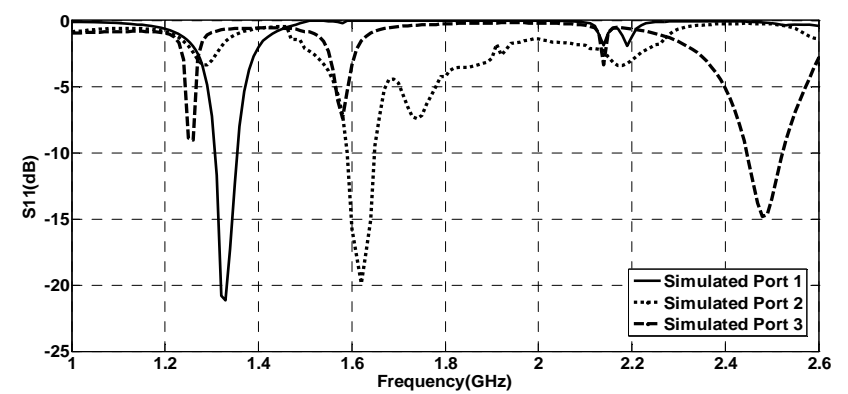




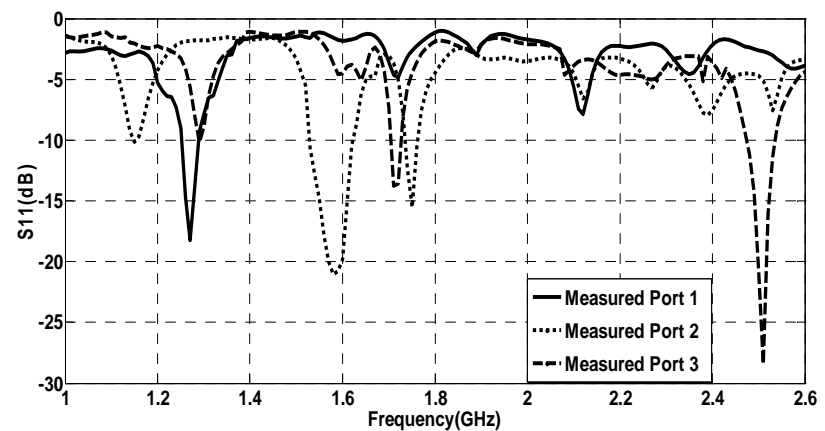

Figure 3. Simulated and measured $S_{11}$ of the proposed stacked tri-band CP antenna
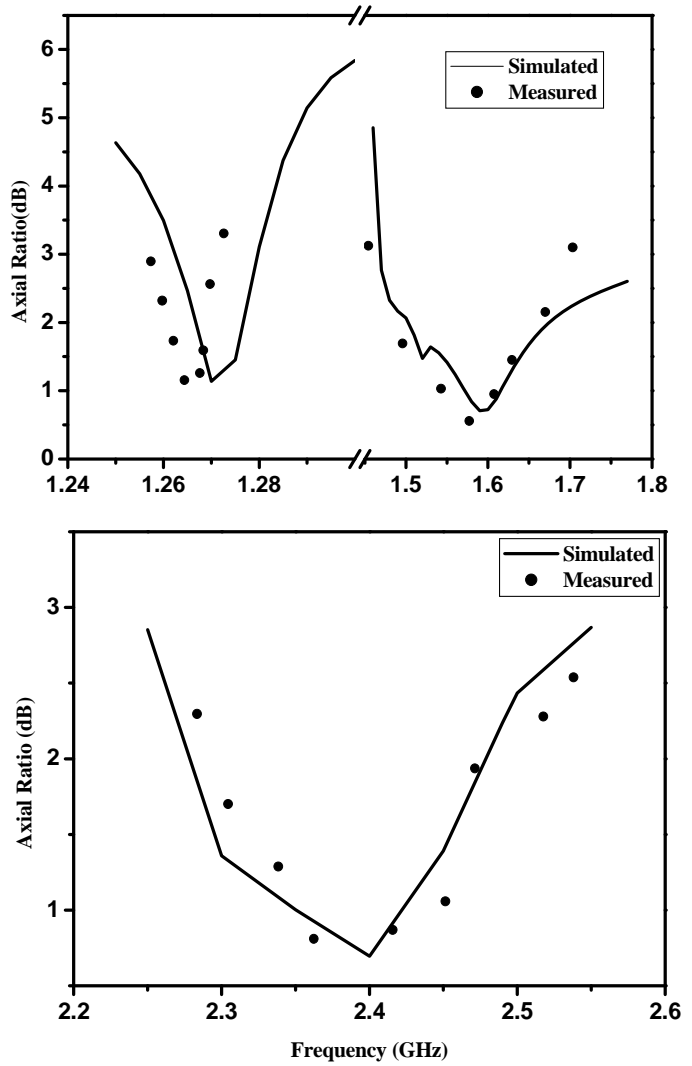

Figure 4. Simulated and measured axis ratio of the proposed stacked triband CP antenna.

The simulated axial ratio against frequency in the broadside direction is shown in Fig. 4. The measured $3 \mathrm{~dB}$ axial ratio bandwidth for the first band is $15 \mathrm{MHz}$, from 1.258 to $1.273 \mathrm{GHz}$ for RHCP, corresponding to about $1.2 \%$ with respect to $1.268 \mathrm{GHz}$ for CNSS B3 application. The measured axial ratio bandwidth for the second band is $180 \mathrm{MHz}$, from 1.5 to $1.68 \mathrm{GHz}$ for LHCP, corresponding to about $11 \%$ with respect to $1.615 \mathrm{GHz}$ for CNSS L band, and the measured axial ratio bandwidth for the third band is 240 $\mathrm{MHz}$, from 2.28 to $2.52 \mathrm{GHz}$ for RHCP, corresponding to about $9.6 \%$ with respect to $2.49 \mathrm{GHz}$ for CNSS application.

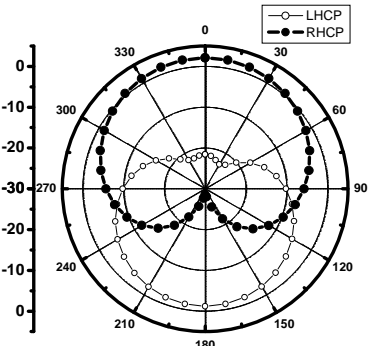

(a)

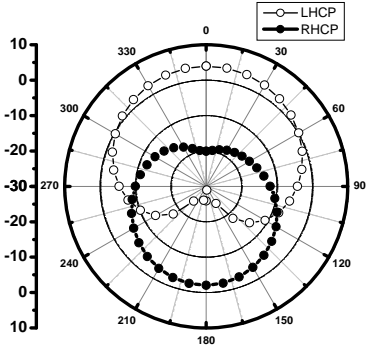

(c)

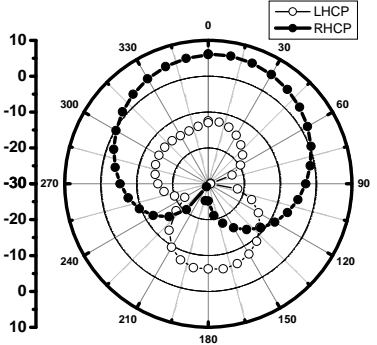

(e)

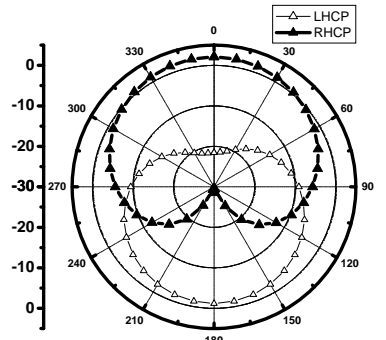

(b)

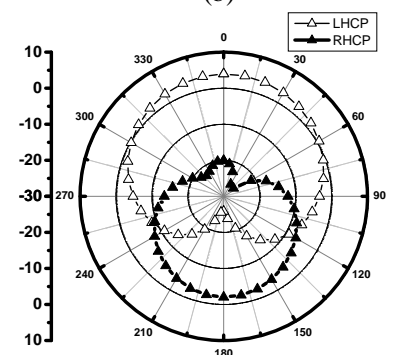

(d)

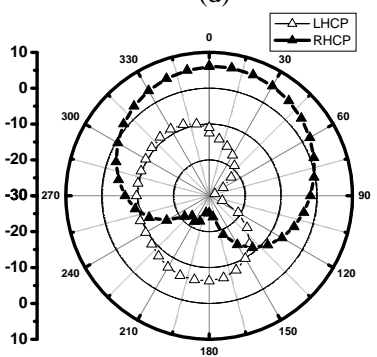

(f)
Figure 5. Measured radiation pattern: (a) XoZ plane at $1.268 \mathrm{GHz}$, (b) YoZ plane at $1.268 \mathrm{GHz}$, (c) XoZ plane at $1.615 \mathrm{GHz}$, (d) YoZ plane at $1.615 \mathrm{GHz}$, (e) XoZ plane at $2.49 \mathrm{GHz}$, (f) YoZ plane at $2.49 \mathrm{GHz}$.

Fig. 5 show the measured radiation patterns in XoZ-plane and YoZ-plane at three frequency. According to the measured radiation patterns, RHCP radiation patterns at the low and high band and LHCP radiation patterns at the second band are excited in respectively. The radiation patterns are symmetrical at $1.268 \mathrm{GHz}$ for the XoZ-plane with a $3-\mathrm{dB}$ beamwidth of $96^{\circ}$ and the YoZ-plane with a beamwidth of $92^{\circ}$. At the second and third bands, the beamwidth is narrower than that of the first band. Also, the radiation patterns are tilted slightly at the high frequency bands. The measured gain values at the all three bands of the proposed antenna are $3.0 \mathrm{dBi}$ at $1.268 \mathrm{GHz}, 3.95 \mathrm{dBi}$ at $1.615 \mathrm{GHz}$ and $6.3 \mathrm{dBi}$ at $2.49 \mathrm{GHz}$, respectively.

\section{CONCLUSION}

A novel triple-band circularly polarized stacked patch antenna is proposed in this paper and fabricated. The proposed antenna can be used in CNSS systems, since its bands of operation are: $1.268 \mathrm{GHz}, 1.615 \mathrm{GHz}$ and $2.49 \mathrm{GHz}$. The radiation is circularly polarized at each band. For the low and high bands the wave is RHCP, while for the medium band it is LHCP. The broad beamwidth , small size 
and high gain of this antenna makes it suitable for the CNSS applications.

\section{REFERENCES}

[1] G. Ramesh, P. Bhartia, I. Bahl, and A. Ittipiboon, Microstrip Antenna Design Handbook. London, U.K.: Artech House, 2001, pp. 493-526.

[2] W. S. Chen, C. K. Wu, and K. L. Wong, "Single-feed square-ring microstrip antenna with truncated corners for compact circular polarization operation,” Electron Lett., vol. 34, pp. 1045-1047, May 1998.

[3] Z. B Wang, S. J Fang, S. Q Fu, and S. W Lv, "Dual-band probe-fed stacked patch antenna for GNSS applications", IEEE Antenna and Wireless Propogation Letters, pp.100-103, Aug 2009.

[4] D. M. Pozar and S. M. Duffy, "A dual-band circularly polarized patch aperture-coupled stacked microstrip antenna for global positioning satellite,” IEEE Trans. Antennas Propag., vol. 45, no. 11, pp.16181625, Nov. 1997.

[5] Nasimuddin, Z. N. Chen, and X. Qing, "Dual-band circularly polarized S-shaped slotted patch antenna with a small frequency- ratio,” IEEE Trans. Antennas Propag., vol. 58, no. 6, pp. 2112-2115, June. 2010.

[6] S. L. Ma and J. S Row, "Design of single-feed Dual-frequency patch antenna for GPS and WLAN applications," IEEE Trans. Antennas Propag., vol. 59, no. 9, pp. 3433-3436, Sep. 2011.

[7] P. Nayeri, K. F. Lee, A. Z. Elsherbeni, and F. Yang, "Dual-band circularly polarized antennas using stacked patches with asymmetric U-slots,” IEEE Antennas Wireless Propag. Lett., vol. 10, pp. 492-495, 2011.

[8] Y. Zhou, C. C. Chen, and J. L. Volakis, "Dual band proximity-fed stacked patch antenna for tri-band GPS applications,” IEEE Trans. Antennas Propag., vol. 55, no. 1, pp. 220-223, 2007.

[9] O. P. Falade, M. U. Rehman, Y. Gao, X. D. Chen, and C. G. Parini, Single feed stacked patch circular polarized antenna for triple band GPS receivers, I IEEE Trans. Antennas Propag, vol. 60, no. 11, pp.4479-4484,october, 2012

[10] W. Liao, Q. X. Chu, and S. Du, "Tri-band circularly polarized stacked microstrip antenna for GPS and CNSS applications," in Proc. ICMMT,2010, pp. 252-255. 